\title{
Bieberbach's Conjecture, the de Branges and Weinstein Functions and the Askey-Gasper Inequality
}

\author{
Wolfram Koepf \\ This article is dedicated to Dick Askey on occasion of his seventieth birthday
}

\begin{abstract}
The Bieberbach conjecture about the coefficients of univalent functions of the unit disk was formulated by Ludwig Bieberbach in 1916 [Bieberbach1916]. The conjecture states that the coefficients of univalent functions are majorized by those of the Koebe function which maps the unit disk onto a radially slit plane.

The Bieberbach conjecture was quite a difficult problem, and it was surprisingly proved by Louis de Branges in 1984 [deBranges 1985] when some experts were rather trying to disprove it. It turned out that an inequality of Askey and Gasper [AskeyGasper1976] about certain hypergeometric functions played a crucial role in de Branges' proof.

In this article I describe the historical development of the conjecture and the main ideas that led to the proof. The proof of Lenard Weinstein (1991) [Weinstein1991] follows, and it is shown how the two proofs are interrelated.

Both proofs depend on polynomial systems that are directly related with the Koebe function. At this point algorithms of computer algebra come into the play, and computer demonstrations are given that show how important parts of the proofs can be automated.
\end{abstract}

\section{The Bieberbach Conjecture}

The origin of the Bieberbach conjecture goes back to the middle of the nineteenth century when the Riemann Mapping Theorem was formulated

1851 RIEMANN Mapping theorem

The Riemann mapping theorem states that for every simply connected domain $G \subset \widehat{\mathbb{C}}$ of the extended complex plane $\widehat{\mathbb{C}}$ there is a univalent and meromorphic function $f: \mathbb{D} \rightarrow G(\mathbb{D}:=$ $\{z \in \mathbb{C}|| z \mid<1\}$ ). Since univalent functions don't possess zero derivatives, there is no loss of generality to assume that $f$ is normalized by $f(0)=0$ and $f^{\prime}(0)=1$ and therefore an element of

$$
S:=\left\{f: \mathbb{D} \rightarrow \mathbb{C} \mid f(z)=z+a_{2} z^{2}+a_{3} z^{3}+\ldots \text { analytic and one-to-one }\right\} .
$$

Another important family is given by $(\Delta:=\{z \in \widehat{\mathbb{C}}|| z \mid>1\})$

$$
\Sigma:=\left\{g: \Delta \rightarrow \widehat{\mathbb{C}} \mid g(z)=z+\frac{b_{1}}{z}+\frac{b_{2}}{z^{2}}+\ldots \text { meromorphic and one-to-one }\right\} .
$$

Riemann's proof of the Riemann mapping theorem was incomplete. Carathéodory [Carathéodory1912] and Koebe [Koebe1912] proved the mapping theorem at the beginning of the twentieth century. 


\section{CARATHÉODORY Proof of the \\ KOEBE mapping theorem}

Moreover, Koebe showed that $S$ is compact w.r.t. the topology of locally uniform convergence ([Koebe1907], [Koebe1909]).

$1909 \quad$ KoEBE $\quad$ Compactness of $S$

Hence, since $a_{n}$ is a continuous functional,

$$
k_{n}:=\max _{f \in S}\left|a_{n}(f)\right|
$$

exists.

Soon later Gronwall received a first result w.r.t. the coefficient problem [Gronwall1914].

1914 Gronwall Area theorem in $\Sigma$

The Area Theorem is an inequality that expresses the obvious fact that the area of the complement of the range of a function $g(z)=z+\frac{b_{1}}{z}+\frac{b_{2}}{z^{2}}+\ldots \in \Sigma$ is non-negative

$$
\sum_{n=1}^{\infty} n\left|b_{n}\right|^{2} \leq 1
$$

Without knowing Gronwall's work, Bieberbach proved the same relation, and received the first coefficient result within $S$ [Bieberbach1916].

$1916 \quad$ BIEBERBACH $\quad\left|a_{2}\right| \leq 2$

This result is deduced from the relation $\left|b_{1}\right| \leq 1$, which is a consequence of (1), by a simple transformation to $S$. Since by (1) $\left|b_{1}\right|=1$ implies $b_{2}=b_{3}=\cdots=0$, it follows that $a_{2}=2$ occurs if and only if $f$ is the Koebe function

$$
K(z):=\frac{z}{(1-z)^{2}}=\frac{1}{4}\left(\left(\frac{1+z}{1-z}\right)^{2}-1\right)=\sum_{n=1}^{\infty} n z^{n},
$$

whose image domain is a plane that is radially slit on the negative real axis. In a footnote Bieberbach wrote "Vielleicht ist überhaupt $k_{n}=n . "$ This statement was called the Bieberbach Conjecture.

$1916 \quad$ CONJECture: $\quad\left|a_{n}\right| \leq n$

The methods that were accessible at those times seemed not to be strong enough to prove this assertion in its completeness. Hence one tried to find partial solutions, i.e.

1. to prove the Bieberbach conjecture under further geometrical conditions for the image domains, or

\footnotetext{
${ }^{1}$ Perhaps $k_{n}=n$ is generally valid.
} 
2. to solve the problem for specific values of $n$, e.g. for $n=3$ or $n=4$.

The first progress for one of these questions was given by Charles Loewner [Loewner1917].

\begin{tabular}{|ll|}
\hline $1917 \quad$ LOEWNER & $\left|a_{n}\right| \leq 1$ \\
& (convex functions) \\
\hline
\end{tabular}

A univalent function is called convex if its image domain is convex.

If we consider only domains that are surrounded by polygons with vertices $w_{k} \in \mathbb{C}$ and interior angles $\alpha_{k} \pi(k=1, \ldots, n)$, then the Schwarz reflection principle implies the SchwarzChristoffel Formula for the mapping function $f$

$$
1+z \frac{f^{\prime \prime}}{f^{\prime}}(z)=\sum_{k=1}^{n} \frac{1-\alpha_{k}}{2} \cdot \frac{1+\overline{f^{-1}\left(w_{k}\right)} z}{1-\overline{f^{-1}\left(w_{k}\right)} z}
$$

that represents a functional differential equation which in general cannot be solved explicitly, but since

$$
\sum_{k=1}^{n} \frac{1-\alpha_{k}}{2}=1
$$

(the rotation of the boundary for one cycle is $2 \pi$ ) and

$$
\left|f^{-1}\left(w_{k}\right)\right|=1 \quad(k=1, \ldots, n)
$$

(the preimages of the vertices lie on $\partial \mathbb{D}$ ) it follows that

$$
\operatorname{Re}\left(1+z \frac{f^{\prime \prime}}{f^{\prime}}(z)\right)>0 \quad(z \in \mathbb{D})
$$

if $\alpha_{k}<1$ for all $k=1, \ldots, n$, which corresponds exactly to the convex case. Moreover, it is easy to prove that (2) implies the univalence and convexity of $f$ (see e.g. [Study1913]).

An elementary estimate which goes back to Carathéodory ([Carathéodory1907], [Carathéodory1911], 1907) and which is valid for functions with positive real part

$$
\operatorname{Re}\left(1+\sum_{n=1}^{\infty} p_{n} z^{n}\right)>0 \Longrightarrow\left|p_{n}\right| \leq 2(n \in \mathbb{N}:=\{1,2,3, \ldots\})
$$

enables an induction proof for the inequalities $\left|a_{n}\right| \leq 1 \quad(n \in \mathbb{N})$ for convex functions. This result analytically expresses the fact that convex domains are far away from being extremal w.r.t. the entirety of all simply connected domains.

The next step towards the Bieberbach conjecture was given by R. Nevanlinna [Nevanlinna1920].

\begin{tabular}{|ll|}
\hline $1920 \quad$ NeVAnLinNa & $\left|a_{n}\right| \leq n$ \\
& (starlike functions) \\
\hline
\end{tabular}

He considered univalent functions with an image domain that is starlike w.r.t. the origin. The proof of the Bieberbach conjecture for starlike functions is also given by an analytic representation. First one proves with the aid of Schwarz' lemma that the starlikeness of $f(\mathbb{D})$ implies 
the starlikeness of $f\left(\mathbb{D}_{r}\right)\left(\mathbb{D}_{r}:=\{z \in \mathbb{C}|| z \mid<r\}\right)$ for all $r \in(0,1)$. Next, by geometrical considerations it follows that $\left|\arg z f^{\prime}(z)-\arg f(z)\right|<\frac{\pi}{2}$, hence

$$
\operatorname{Re}\left(z \frac{f^{\prime}}{f}(z)\right)>0 \quad(z \in \mathbb{D}) .
$$

Carathéodory's estimate (3) for functions with positive real part yields the result. Again, one can prove that Equation (4) implies the univalence and starlikeness of $f$.

From Nevanlinna's result it became clear that there was some chance for the Bieberbach conjecture to be true since it was true for a rather large subset of $S$. Nevertheless, it was not yet known whether the Bieberbach conjecture was true for a single $n>2$. This step was finished by Loewner [Loewner1923], again. Maybe Loewner's article was the most important step towards the proof of the Bieberbach conjecture, at least it was the first decisive one.

\begin{tabular}{||ll||}
\hline $1923 \quad$ LOEWNER & $\left|a_{3}\right| \leq 3$ \\
& (Loewner theory) \\
\hline
\end{tabular}

Loewner gave an analytic representation for a subset of $S$ which is dense w.r.t. the topology of locally uniform convergence. Loewner's theorem states that for every $f$ of this dense subset of $S$ there is a Loewner chain, i.e. a family of functions $\{f(z, t) \mid t \geq 0\}$ with

$$
f(z, t)=e^{t} z+\sum_{n=2}^{\infty} a_{n}(t) z^{n}, \quad\left(z \in \mathbb{D}, t \geq 0, a_{n}(t) \in \mathbb{C}(n \geq 2)\right),
$$

which start with $f$

$$
f(z, 0)=f(z)
$$

and for which the relation

$$
\operatorname{Re}\left(\frac{\dot{f}(z, t)}{z f^{\prime}(z, t)}\right)>0 \quad(z \in \mathbb{D})
$$

is satisfied. Here ' and denotes the partial derivatives w.r.t $z$ and $t$, respectively, as usual. The above equation is referred to as the Loewner differential equation. It geometrically states that - as a result of Schwarz' lemma, again - the image domains of $f(\mathbb{D}, t)$ expand as $t$ increases. Hence Loewner investigated the dynamics of univalent functions. The subset of $S$ that he considered consists of those functions whose image domains are all of $\mathbb{C}$ besides a slit which is a Jordan arc. In this case, the Loewner chain consists of those functions whose slit gets shorter as $t$ increases.

The Loewner differential equation characterizes univalent functions, since it turns out that for all $f \in S$ there is a Loewner chain. Furthermore, one can prove that under rather general conditions for the function

$$
p(z, t):=\frac{\dot{f}(z, t)}{z f^{\prime}(z, t)}
$$

the univalence of $f(z, 0)$ implies the univalence of $f(z, t) \quad(t>0)$.

Very easily it follows from (5) that $\left|a_{3}\right| \leq 3$ in $S$ (see e.g. [Duren1983], $\S 3.5$ ). But only in 1973 Nehari succeeded in proving $\left|a_{4}\right| \leq 4$ using exclusively Loewner's theory.

Next, Littlewood could prove that the Bieberbach conjecture gives the correct order of magnitude for the coefficient growth [Littlewood1925]. 


\section{$1925 \quad$ LITTLEWOOD $\quad\left|a_{n}\right|<e \cdot n$}

If $f \in S$, then the function $h$ with $\frac{h(z)}{z}:=\sqrt{\frac{f\left(z^{2}\right)}{z^{2}}}$, which is called the square root transform of $f$, is an odd univalent function and vice versa. By $S_{\text {odd }} \subset S$ we denote the set of odd univalent functions.

From the Koebe distortion theorem $|f(z)| \leq \frac{|z|}{(1-|z|)^{2}}$, which was proved by Bieberbach [Bieberbach1916], it follows that $|h(z)| \leq \frac{|z|}{1-|z|^{2}}$ and therefore

$$
\text { area }\left(h\left(\mathbb{D}_{r}\right)\right) \leq \pi \frac{r^{2}}{\left(1-r^{2}\right)^{2}} .
$$

From this, by Parseval's theorem it follows that

$$
\frac{1}{2 \pi} \int_{0}^{2 \pi}\left|f\left(r^{2} e^{i \theta}\right)\right| d \theta=\frac{1}{4 \pi} \int_{0}^{4 \pi}\left|f\left(r^{2} e^{i \theta}\right)\right| d \theta=\frac{1}{2 \pi} \int_{0}^{2 \pi}\left|f\left(r^{2} e^{2 i \theta}\right)\right| d \theta=\frac{1}{2 \pi} \int_{0}^{2 \pi}\left|h\left(r e^{i \theta}\right)\right|^{2} d \theta \leq \frac{r^{2}}{1-r^{2}} .
$$

Furthermore, the Cauchy integral formula gives

$$
\left|a_{n}\right| \leq \frac{1}{r^{n}}\left(\frac{1}{2 \pi} \int_{0}^{2 \pi}\left|f\left(r e^{i \theta}\right)\right| d \theta\right) \leq \frac{1}{r^{n-1}(1-r)}=\left(1+\frac{1}{n-1}\right)^{n-1} \cdot n
$$

using $r:=1-\frac{1}{n}$. Since $\left(1+\frac{1}{n}\right)^{n}$ increases towards $e$, Littlewood's result follows.

This method has the disadvantage that by taking an integral mean information is thrown away so that a sharp result such as the Bieberbach conjecture is not accessible.

About a decade after the formulation of the Bieberbach conjecture it was therefore proved

1. for an interesting subclass,

2. for the third coefficient and

3. in its order of magnitude.

As next step the Bieberbach conjecture could be verified for another subclass of $S$ : for univalent functions with real Taylor coefficients ([Dieudonné1931], [Rogosinski1932]). These are characterized by domains that are symmetric w.r.t. the real axis.

\begin{tabular}{|lll}
1931 & DIEUdONNÉ & $\left|a_{n}\right| \leq n$ \\
& ROgOSINSKI & (real coefficients)
\end{tabular}

Both authors used quite different methods: the question seemed to be in the air.

\section{The Littlewood-Paley and the Robertson Conjecture}

Again by the integral mean method Littlewood and Paley [LP1932] could show that for odd univalent functions $h(z)=\sum_{n=1}^{\infty} c_{n} z^{n} \in S_{\text {odd }}$

$$
\left|c_{n}\right| \leq A
$$


for a constant $A$ that does neither depend on $h$ nor on $n$. Their article contains the footnote "No doubt the true bound is given by $A=1$." Hence they conjectured that the square root transform of the Koebe function $\frac{z}{1-z^{2}}=\sum_{n=0}^{\infty} z^{2 n+1}$ is extremal for the given problem.

\begin{tabular}{|lll|}
\hline 1932 & $\begin{array}{l}\text { LiTtLEWOOD } \\
\text { PALEY }\end{array}$ & $\begin{array}{l}\text { Littlewood-Paley conjecture } \\
\text { (odd functions) }\end{array}$ \\
\hline
\end{tabular}

Instantaneously Fekete and Szegö [FS1933] disproved the Littlewood-Paley Conjecture by using Loewner's method. This was the first relapse in the history of the Bieberbach conjecture.

1933 \begin{tabular}{ll|} 
FEKETE \\
SZEGÖ
\end{tabular}$\quad \max _{h \in S_{\text {odd }}}\left|c_{5}\right|=\frac{1}{2}+e^{-2 / 3}=1.0134 \ldots>1$

The Littlewood-Paley conjecture would have implied the Bieberbach conjecture. Assume $h(z)=$ $\sum_{n=1}^{\infty} c_{n} z^{n}$ is the square root transform of $f(z)=\sum_{n=1}^{\infty} a_{n} z^{n}$, then

$$
a_{n}=\sum_{k=1}^{n} c_{2(n-k)+1} \cdot c_{2 k-1}
$$

so that obviously $\left|c_{n}\right| \leq 1 \quad(n \in \mathbb{N})$ implies the Bieberbach conjecture.

Even though the Littlewood-Paley conjecture turned out to be false, it showed the way in the correct direction. Robertson [Robertson1936] conjectured a somewhat weaker condition which, nevertheless, implies the Bieberbach conjecture.

\begin{tabular}{||ll||}
\hline $1936 \quad$ RoBERTSON & Robertson conjecture \\
\hline
\end{tabular}

Robertson's Conjecture states for odd univalent functions the inequality

$$
h \in S_{\text {odd }} \quad \Longrightarrow \quad \sum_{k=1}^{n}\left|c_{2 k-1}\right|^{2} \leq n,
$$

which by an application of the Cauchy-Schwarz inequality to (7) implies the Bieberbach conjecture. Robertson showed the Robertson conjecture for $n=3$.

This work should turn out to be the second decisive step towards the proof of the Bieberbach conjecture. Robertson's essential new idea was to consider weighted quadratic means of Taylor coefficients.

At the end of the 1930s Schiffer [Schiffer1938] developed a variational method that was suitable to solve extremal problems within $S$. Whereas elsewhere in variational calculus one of the major concerns is the question of existence of a solution, in our case existence is not an issue since $S$ is compact. Instead it is rather difficult to find suitable comparison functions since $S$ is everything else but a linear space.

\begin{tabular}{|ll|}
\hline $1938 \quad$ SCHIFFER $\quad$ boundary variation \\
\hline
\end{tabular}


The idea behind Schiffer's boundary variation is to compose a univalent function $f$ with a family of in $f(\mathbb{D})$ almost identical mappings. The conclusion is a differential equation that the extremal function characterizes. Let $L$ be a continuous linear functional, and let $\operatorname{Re} L\left(f_{0}\right)=\max _{f \in S} \operatorname{Re} L(f)$, then as a-rather complicated-result of Schiffer's variational method it turns out that $f_{0}(\mathbb{D})=$ $\mathbb{C} \backslash \Gamma$, where $\Gamma$ is an analytic arc satisfying the differential functional equation

$$
\frac{1}{\Gamma(t)^{2}} L\left(\frac{f^{2}}{f-\Gamma(t)}\right) d \Gamma(t)^{2}>0,
$$

hence $\Gamma$ is the trajectory of a quadratic differential. An application of the Schwarz reflection principle for the coefficient functional $L=\operatorname{Re} a_{n}(f)$ yields for the mapping function $f$ the Schiffer differential equation

$$
\left(\frac{z f^{\prime}(z)}{f(z)}\right)^{2} P_{n}\left(\frac{1}{f(z)}\right)=R_{n}(z),
$$

where $P_{n}$ is the polynomial of degree $n-1$ with the generating function

$$
\frac{\zeta(f(z))^{2}}{1-\zeta f(z)}=\sum_{k=2}^{\infty} P_{k}(\zeta) z^{k}
$$

and $R_{n}$ is the rational function given by

$$
R_{n}(z)=(n-1) a_{n}+\sum_{k=1}^{n-1}\left(k a_{k} z^{k-n}+k \overline{a_{k}} z^{n-k}\right) .
$$

Hence every solution $f$ of Bieberbach's coefficient problem must also satisfy the Schiffer differential equation. In particular, $f(\mathbb{D})=\mathbb{C} \backslash \Gamma$ and $\Gamma$ is an analytic arc. For every $n \geq 2$ the Koebe function is a solution of the Schiffer differential equation, but unfortunately the solution is not unique.

Eventually in 1955 Garabedian and Schiffer [GS1955] were able to use Schiffer's variational method to prove the Bieberbach conjecture for $n=4$.

In contrast to Schiffer's variational method the Grunsky inequalities [Grunsky1939] are rather elementary. They generalize Gronwall's area theorem.

\section{GRUNSKY Grunsky inequalities}

For all $g \in \Sigma$ let

$$
\ln \frac{g(z)-g(\zeta)}{z-\zeta}=:-\sum_{n=1}^{\infty} \sum_{k=1}^{\infty} \gamma_{n k} z^{-n} \zeta^{-k} \quad(z, \zeta \in \Delta) .
$$

The Grunsky coefficients $\gamma_{n k}$ are polynomials in the coefficients $b_{n}$ of $g$. Note that a representation of the form (8) for a meromorphic function $g(z)=z+\frac{b_{1}}{z}+\frac{b_{2}}{z^{2}}+\ldots$ exists if and only if $g \in \Sigma$.

The Grunsky inequalities

$$
\left|\sum_{n=1}^{N} \sum_{k=1}^{N} \gamma_{n k} \lambda_{n} \mu_{k}\right| \leq \sum_{n=1}^{N} \frac{\left|\lambda_{n}\right|^{2}}{n} \cdot \sum_{k=1}^{N} \frac{\left|\mu_{k}\right|^{2}}{k}
$$


are valid for all $N \in \mathbb{N}$ and $\lambda_{n}, \mu_{n} \in \mathbb{C}(n=1, \ldots, N)$.

Surprisingly, they were used for a very elementary and simple proof of the Bieberbach conjecture for $n=4$ by Charzyński and Schiffer [CS1960] in 1960. Eight years later Pederson [Pederson1968] and-independently-Ozawa ([Ozawa1969a], [Ozawa1969b]) used the Grunsky method to show $\left|a_{6}\right| \leq 6$ for $f \in S$.

Likewise Friedland [Friedland1970] was able to prove the Robertson conjecture for $n=4$ in 1970.

At the end of the 1930s the interest for the Bieberbach conjecture was abated. The search for a proof had been not successful enough. It took until the 1950s when the problem was reconsidered.

Hayman had been asked by Littlewood to prove the Bieberbach conjecture as PhD topic. Since a full proof seemed to be out of sight, Hayman asked the still difficult question about the behavior of $\frac{\left|a_{n}\right|}{n}$ for $n \rightarrow \infty$, and he could settle it [Hayman1955].

\section{HAYMAN Regularity theorem}

In a first step he gave an elementary proof of the fact that for $f \in S$

$$
\lim _{r \rightarrow 1}\left(1-r^{2}\right) \max _{|z|=r}|f(z)|=\alpha \leq 1 .
$$

The number $\alpha$ is called the Hayman index of $f$. Finally Hayman could show that for every function $f \in S$

$$
\lim _{n \rightarrow \infty} \frac{\left|a_{n}(f)\right|}{n}=\alpha
$$

This is clearly much information since it is not obvious at all that this limit exists for every $f \in S$. On the other hand Hayman's statement does not indicate the Bieberbach conjecture for large $n \in \mathbb{N}$ since the speed of convergence considerably depends on $f$. Hayman's result might give some evidence for the truth of the Bieberbach conjecture, but this is clearly a fallacy, since the regularity theorem implies for odd univalent functions $h(z)=\sum_{n=1}^{\infty} c_{n} z^{n} \in S_{\text {odd }}$ with $\frac{h(z)}{z}=\sqrt{\frac{f\left(z^{2}\right)}{z^{2}}}$ the relation

$$
\lim _{n \rightarrow \infty}\left|c_{2 n+1}\right|=\sqrt{\alpha} \leq 1
$$

although the Littlewood-Paley conjecture is false!

In the 1950s the Bieberbach conjecture could be proved again for a further subset of $S$, the closeto-convex functions [Reade1955].

\begin{tabular}{|ll|}
\hline $1955 \quad$ READE & $\mid \begin{array}{l}\left|a_{n}\right| \leq n \\
\text { (close-to-convex functions) }\end{array}$ \\
\end{tabular}

A univalent function is called close-to-convex functions if its range $f(\mathbb{D})$ is the complement of a union of rays that are pairwise disjoint. Of course, starlike functions have this property so that Reade's result generalizes Nevanlinna's.

Let us remind the fact that also in 1955 the Bieberbach conjecture was proved the first time for $n=4$. 


$$
1955 \begin{array}{ll}
\text { GaRABEDIAN } \\
\text { SCHIFFER }
\end{array} \quad \begin{aligned}
& \left|a_{4}\right| \leq 4 \\
& \text { (Loewner th., Schiffer var., etc.) }
\end{aligned}
$$

Using the Grunsky inequalities an elementary proof for the same coefficient was given in [CS1960].

\begin{tabular}{|lll|}
\hline 1960 & $\begin{array}{l}\text { CHARZYŃSKI } \\
\text { SCHIFFER }\end{array}$ & $\left|a_{4}\right| \leq 4$ \\
& (Grunsky inequalities) \\
\hline
\end{tabular}

By an ingenious choice of the free parameters in the Grunsky inequalities Milin was able to improve Littlewood's result considerably ([Milin1965], [Milin1971]).

$1965 \quad$ MiLiN $\quad\left|a_{n}\right|<1.243 \cdot n$

\section{The Milin Conjecture}

The researchers in the former soviet union worked also on the last essential ingredient which made de Branges' proof possible. After some decades one knew that it was very difficult to obtain informations about the coefficients $a_{n}(f)$ of univalent functions. The success of the Grunsky inequalities suggested that it should be simpler to obtain results about the coefficients $d_{n}(f)$ of the function

$$
\ln \frac{f(z)}{z}=: \sum_{n=1}^{\infty} d_{n} z^{n} .
$$

These are called the logarithmic coefficients of $f$. The use of the logarithmic coefficients is also suggested by Loewner's method.

N. A. Lebedev and I. M. Milin ([LM1965], [Milin1967], [Milin1971]) worked on the question how to turn information about the logarithmic coefficients into information about the coefficients of $f$ itself. They discovered that it was easier to lift informations about certain quadratic means of coefficients. This could be combined with the Robertson conjecture. They showed rather elementary—almost entirely by the use of the Cauchy-Schwarz inequality: If $\psi(z)=\sum_{k=0}^{\infty} \beta_{k} z^{k}$ with $\beta_{0}=1$ has positive radius of convergence, then the same is true for $\varphi(z):=\ln \psi(z)=$ $\sum_{k=1}^{\infty} \alpha_{k} z^{k}$, and the following relations between the coefficients are valid

$$
\begin{gathered}
\sum_{k=0}^{\infty}\left|\beta_{k}\right|^{2} \leq \exp \left(\sum_{k=1}^{\infty} k\left|\alpha_{k}\right|^{2}\right) \\
\frac{1}{n+1} \sum_{k=0}^{n}\left|\beta_{k}\right|^{2} \leq \exp \left(\frac{1}{n+1} \sum_{k=1}^{n}(n+1-k)\left(k\left|\alpha_{k}\right|^{2}-\frac{1}{k}\right)\right)
\end{gathered}
$$

and

$$
\left|\beta_{n}\right|^{2} \leq \exp \left(\sum_{k=1}^{n}\left(k\left|\alpha_{k}\right|^{2}-\frac{1}{k}\right)\right) .
$$

Note that the Lebedev-Milin inequalities (10)-(12) are completely independent of univalent functions. Hence it was possible to use them to solve quite different function theoretic problems (see e.g. [Aharonov1970], [Nehari1970], [Leung1978], [Leung1979]). 


\begin{tabular}{||ll||}
\hline 1967 & $\begin{array}{l}\text { LEBEDEV } \\
\text { MILIN }\end{array}$ \\
\hline
\end{tabular}

The nature of the Lebedev-Milin inequalities enables their application to the Bieberbach conjecture. Therefore it is necessary that they are suitably sharp for the Koebe function. If we set

$$
\psi(z):=\frac{f(z)}{z}=\sum_{n=0}^{\infty} a_{n+1} z^{n}
$$

then

$$
\varphi(z)=\ln \frac{f(z)}{z}=\sum_{n=1}^{\infty} d_{n} z^{n}
$$

If $f \in S$, then $d_{n}$ are the logarithmic coefficients of $f$. Let further $h(z)=\sum_{n=1}^{\infty} c_{n} z^{n}$ be the square root transform of $f$.

Assume, for some $n \in \mathbb{N}$ the Milin Conjecture

$$
\sum_{k=1}^{n}(n+1-k)\left(k\left|d_{k}\right|^{2}-\frac{4}{k}\right) \leq 0
$$

is valid, then with $\ln \frac{h(z)}{z}=\frac{1}{2} \varphi\left(z^{2}\right)$ it follows from the second Milin inequality that

$$
\frac{1}{n+1} \sum_{k=1}^{n+1}\left|c_{2 k-1}\right|^{2} \leq 1
$$

hence the Robertson conjecture and therefore the Bieberbach conjecture for the index $n+1$.

\begin{tabular}{||ll||}
\hline $1971 \quad$ MiLin & Milin conjecture \\
\hline
\end{tabular}

The Milin conjecture is a statement about a weighted quadratic mean of the logarithmic coefficients or of the coefficients of the function

$$
z \frac{f^{\prime}}{f}(z)=1+z\left(\ln \frac{f(z)}{z}\right)^{\prime}=1+\sum_{n=1}^{\infty} n d_{n} z^{n},
$$

whose modulus is bounded by 2 for starlike functions because of (4) and (3), so that for those functions $\left|d_{k}\right| \leq 2 / k(k \in \mathbb{N})$ and therefore the Milin conjecture is valid.

At the end of the 1960s the use of the Grunsky inequalities succeeded in proving specific instances of the Bieberbach conjecture ([Pederson1968], [Ozawa1969a], [Ozawa1969b], [Friedland1970]).

\begin{tabular}{|lll|}
\hline 1968 & $\begin{array}{l}\text { PEDERSON } \\
\text { OZAWA }\end{array}$ & $\begin{array}{l}\left|a_{6}\right| \leq 6 \\
\text { (Grunsky inequalities) }\end{array}$ \\
\hline 1970 & FRIEDLAND & $\begin{array}{l}\text { Robertson conjecture for } n=4 \\
\text { (Grunsky inequalities) }\end{array}$ \\
\hline
\end{tabular}


Another strategy that was considered since Schiffer's work was to characterize the support points of $S$, i.e. those functions that are solutions of linear extremal problems. From Schiffer's theory it was known that in this case $f(\mathbb{D})=\mathbb{C} \backslash \Gamma$ with an analytic curve $\Gamma$. Was it possible to characterize the support points, maybe they could be even parameterized?

A similar question was considered by Brickman [Brickman1970] who asked for the properties of the extreme points of $S$, i.e. those functions that do not have a proper convex representation within $S$.

\begin{tabular}{|ll|}
\hline $1970 \quad$ BRICKMAN & Extreme points \\
\hline
\end{tabular}

For every linear extremal problem there is an extreme point solution. Brickman found a simple proof of the fact that for extreme points $f(\mathbb{D})=\mathbb{C} \backslash \Gamma$, where $\Gamma$ is a curve with increasing absolute value.

In these days one hoped that such functional analytic methods might eventually solve the Bieberbach conjecture. Ironically at the time of the proof of the Milin conjecture by de Branges it turned out, however, that there are too many extreme and support points to be of value for the solution of the Bieberbach conjecture [Hamilton1988]. On the other hand extreme point theory was applied with great success to subfamilies of $S$ like convex and starlike functions ([BMW1971], see also [Koepf1986] and [Koepf1987]).

Another type of exponentiation of the Grunsky inequalities than the one used by Lebedev and Milin was developed by FitzGerald [FitzGerald1972]. Using this approach he could improve Milin's estimate.

$$
1972 \quad \text { FitzGERALD } \quad\left|a_{n}\right|<\sqrt{\frac{7}{6}} \cdot n=1.0801 \ldots \cdot n
$$

In 1978 Horowitz [Horowitz1978] could refine FitzGerald's argument to deduce the inequality

$$
\left|a_{n}\right|<1.0657 \cdot n \quad(n \in \mathbb{N})
$$

In the same year of FitzGerald's result the last single result was established [PS1972] before the general proof.

$$
1972 \quad \begin{aligned}
& \text { PEDERSON } \\
& \text { SCHIFFER }
\end{aligned} \quad\left|a_{5}\right| \leq 5
$$

Now the Bieberbach conjecture was proved for $n \leq 6$, for close-to-convex functions and functions with real coefficients, and one had Hayman's regularity result (9) as well as the estimate (14). But still there was no idea how to prove the Bieberbach conjecture for general $n$. Maybe the Bieberbach conjecture was false? The disproof of the Littlewood-Paley conjecture by Fekete and Szegö as well as the results of other extremal problems - the value

$$
\max \left\{\left|c_{7}\right| \mid h(z)=\sum_{n=1}^{\infty} c_{n} z^{n} \in S_{\text {odd }}, c_{n} \in \mathbb{R}(n \in \mathbb{N})\right\}=1+\frac{7}{1083}
$$

is also larger than 1 [Leeman1976]— hint the possibility that the Bieberbach conjecture could be slightly wrong for some $n \in \mathbb{N}$. 
Some researchers tried now to disprove the Bieberbach conjecture by finding a numerical counterexample. Hummel reported that at the beginning of the 1980s he was sure that the Bieberbach conjecture would collapse around $n=19$. Hummel had been successful in 1976 to disprove a conjecture about the so-called Gelfer functions [Hummel1976] by finding a numerical counterexample.

In [HH1986] Hayman and Hummel found numerical examples of univalent functions whose coefficients seemed to contradict the Bieberbach conjecture. However, the Bieberbach conjecture could not be disproved since the error estimates of the numerical computations could not be made small enough. Nevertheless, there seemed to be more and more evidence against the Bieberbach conjecture.

\section{The de Branges Theorem}

In the spring of 1984 de Branges sent a book manuscript to well-known specialists on univalent functions whose last chapter contained a proof of the Bieberbach conjecture. But some small errors were discovered, and almost nobody read the 385 page manuscript completely. Every previous announcement of a proof of the Bieberbach conjecture had turned out to contain irrepairable mistakes.

De Branges was invited to the Steklov institute in Leningrad, and he gave lectures about his proof in the function theory seminar, one of whose members was Milin, and in June 1984 the members of the seminar were convinced that de Branges' proof was correct! Emel'yanov had translated the essential ideas into Russian language, and Milin worked out the function theoretic nucleus of de Branges' argument and sent out this version which found world-wide distribution [Milin1984].

\begin{tabular}{ll}
1984 DE BRANGES & $\begin{array}{l}\text { Proof of the Milin conjecture, } \\
\text { Robertson conjecture and Bieber- } \\
\text { bach conjecture }\end{array}$ \\
\hline
\end{tabular}

The initial idea of de Branges was the following: Both the Robertson conjecture and the Milin conjecture were statements about quadratic means of coefficients of analytic functions. They could be looked at as statements about the norm in certain Hilbert spaces of analytic functions. This functional analytic approach led him to the following considerations: To prove the Milin conjecture (13) for some $n \in \mathbb{N}$, set

$$
\psi(t):=\sum_{k=1}^{n} \tau_{k}^{n}(t)\left(k\left|d_{k}(t)\right|^{2}-\frac{4}{k}\right),
$$

where the function system $\left(\tau_{k}^{n}\right)_{k=1, \ldots, n}$ of functions $\tau_{k}^{n}: \mathbb{R}_{\geq 0} \rightarrow \mathbb{R}$ is still unknown and will be determined later, and let $d_{k}(t)$ denote the logarithmic coefficients of $e^{-t} f(z, t)$, where $f(z, t)$ is a Loewner chain of $f$.

The relation $\psi(0) \leq 0$ is equivalent to Milin's conjecture if we set

$$
\tau_{k}^{n}(0)=n+1-k \quad(k=1, \ldots, n) .
$$

De Branges succeeded to prove that the Milin conjecture for the index $n \geq 1$ was true if the function system $\left(\tau_{k}^{n}\right)_{k=1, \ldots, n}$ has the properties

$$
\tau_{k}^{n}(t)-\tau_{k+1}^{n}(t)=-\frac{\dot{\tau}_{k}^{n}(t)}{k}-\frac{\dot{\tau}_{k+1}^{n}(t)}{k+1} \quad(k=1, \ldots, n)
$$


as well as

$$
\lim _{t \rightarrow \infty} \tau_{k}^{n}(t)=0 \quad(k=1, \ldots, n)
$$

and

$$
\dot{\tau_{k}^{n}}(t) \leqq 0 \quad\left(t \in \mathbb{R}_{\geqq 0}\right) .
$$

Using these equations and Loewner's differential equation (5) leads-after a lengthy but not difficult computation - to the relation $\dot{\psi}(t) \geq 0$ and therefore to $\psi(0)=-\int_{0}^{\infty} \dot{\psi}(t) d t \leq 0$.

Hence the Bieberbach conjecture for the index $n+1$ was reduced to the existence of the functions $\left(\tau_{k}^{n}\right)_{k=1, \ldots, n}$ with the properties (15)-(18).

Of course the coupled system of differential equations (16) with the initial values (15) has a unique solution, hence the properties (17) and (18) must be fulfilled additionally! Here, de Branges was lucky. Whereas (17) is an easy consequence of the theory of ordinary differential equations, relation (18) is a deep theorem. De Branges gave the explicit representations

$$
\tau_{k}^{n}(t)=e^{-k t}\left(\begin{array}{c}
n+k+1 \\
2 k+1
\end{array}\right){ }_{4} F_{3}\left(\begin{array}{c}
k+\frac{1}{2}, n+k+2, k, k-n \\
k+1,2 k+1, k+\frac{3}{2}
\end{array} \mid e^{-t}\right),
$$

and

$$
\dot{\tau}_{k}^{n}(t)=-k e^{-k t}\left(\begin{array}{c}
n+k+1 \\
1+2 k
\end{array}\right){ }_{3} F_{2}\left(\begin{array}{c}
k+\frac{1}{2}, k-n, n+k+2 \\
2 k+1, k+\frac{3}{2}
\end{array} \mid e^{-t}\right)
$$

of his functions where the power series

$$
{ }_{p} F_{q}\left(\begin{array}{c}
a_{1}, \cdots, a_{p} \\
b_{1}, \ldots, b_{q}
\end{array} \mid y\right)=\sum_{k=0}^{\infty} a_{k}=\sum_{k=0}^{\infty} \frac{\left(a_{1}\right)_{k} \cdots\left(a_{p}\right)_{k}}{\left(b_{1}\right)_{k} \cdots\left(b_{q}\right)_{k}} \frac{y^{k}}{k !}
$$

whose summands $a_{k}$ have a rational term ratio

$$
\frac{a_{k+1}}{a_{k}}=\frac{\left(k+a_{1}\right) \cdots\left(k+a_{p}\right)}{\left(k+b_{1}\right) \cdots\left(k+b_{q}\right)} \cdot \frac{y}{k+1},
$$

as usual denotes the generalized hypergeometric function (see e.g. [Koepf1998]). The summands $a_{k}$ of the hypergeometric function are called hypergeometric terms, and $(a)_{k}=a(a+1) \cdots(a+$ $k-1)$ is the Pochhammer symbol or shifted factorial.

Note that $\dot{\tau}_{k}^{n}$ are polynomials w.r.t the variable $y=e^{-t}$. De Branges verified (18) by hand computations up to $n=5$, hence he reproved the Bieberbach conjecture up to $n=6$ again! Next, he asked his colleague Walter Gautschi from Purdue University to verify the inequality (18) numerically, which was done for $n \leqq 30$, and de Branges became confident of the validity of the general statement.

Note, however, although Gautschi (under several methods) used Sturm sequences (for details, see [Gautschi1986]), because of the oscillatory nature of $\dot{\tau}_{k}^{n}$ (see Figure 1) his numerical computations had to be very careful to obtain correct results. Nowadays, we can apply Sturm sequences or more efficient approaches [CK1992] in a computer algebra system and count the roots easily by rational arithmetic to obtain correct countings since the input polynomials have rational coefficients. In this way Milin's conjecture for $n \leqq 30$, e.g., is easily checked within seconds with a $\mathrm{PC}$ of these days. 


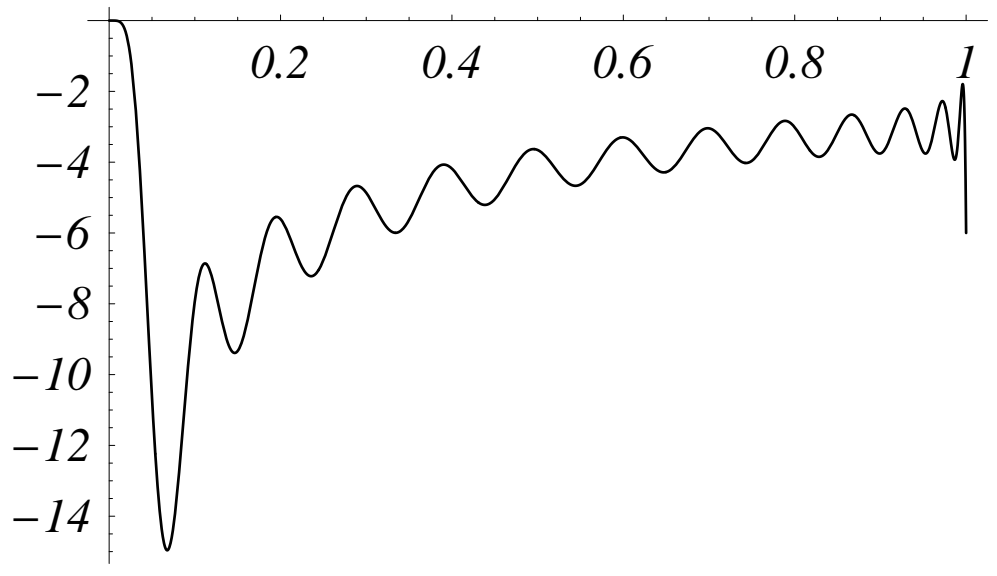

Figure 1: $\dot{\tau}_{6}^{30}(y)$ shows the highly oscillatory character of the derivatives of the de Branges functions

By a phone call of Walter Gautschi with Dick Askey, de Branges finally realized that his relation (18) had been proved by Askey and Gasper not long before, namely in 1976 [AskeyGasper1976]. This finished the proof of the Milin conjecture [deBranges1985].

FitzGerald and Pommerenke [FP1985] realized that the proof was independent of functional analysis and published a purely function theoretic version.

How did Askey and Gasper find out that

$$
{ }_{3} F_{2}\left(\begin{array}{c}
k+\frac{1}{2}, k-n, n+k+2 \\
2 k+1, k+\frac{3}{2}
\end{array} \mid y\right) \geqq 0 ?
$$

This is a consequence of the so-called Askey-Gasper inequality that was proved by detecting ingeniously the Askey-Gasper identity

$$
\begin{gathered}
\frac{(\alpha+2)_{n}}{n !} \cdot{ }_{3} F_{2}\left(\begin{array}{r}
-n, n+2+\alpha, \frac{\alpha+1}{2} \\
\alpha+1, \frac{\alpha+3}{2}
\end{array} \mid x\right)= \\
\sum_{j=0}^{\left\lfloor\frac{n-k}{2}\right\rfloor} \frac{\left(\frac{1}{2}\right)_{j}\left(\frac{\alpha}{2}+1\right)_{n-j}\left(\frac{\alpha+3}{2}\right)_{n-2 j}(\alpha+1)_{n-2 j}}{j !\left(\frac{\alpha+3}{2}\right)_{n-j}\left(\frac{\alpha+1}{2}\right)_{n-2 j}(n-2 j) !} \cdot{ }_{3} F_{2}\left(\begin{array}{c}
2 j-n, n-2 j+\alpha+1, \frac{\alpha+1}{2} \mid \\
\alpha+1, \frac{\alpha+2}{2}
\end{array}\right)
\end{gathered}
$$

which (for $\alpha=2 k$ ) represents the de Branges functions as a linear combination of ${ }_{3} F_{2}$ functions with positive coefficients.

Using Zeilberger's algorithm which was developed in 1990 [Zeilberger1990], computer calculations can prove the Askey-Gasper identity easily [Koepf1998]. Therefore we load the Maple package hsum . mpl which is part of the book [Koepf1998] and includes Zeilberger's algorithm:

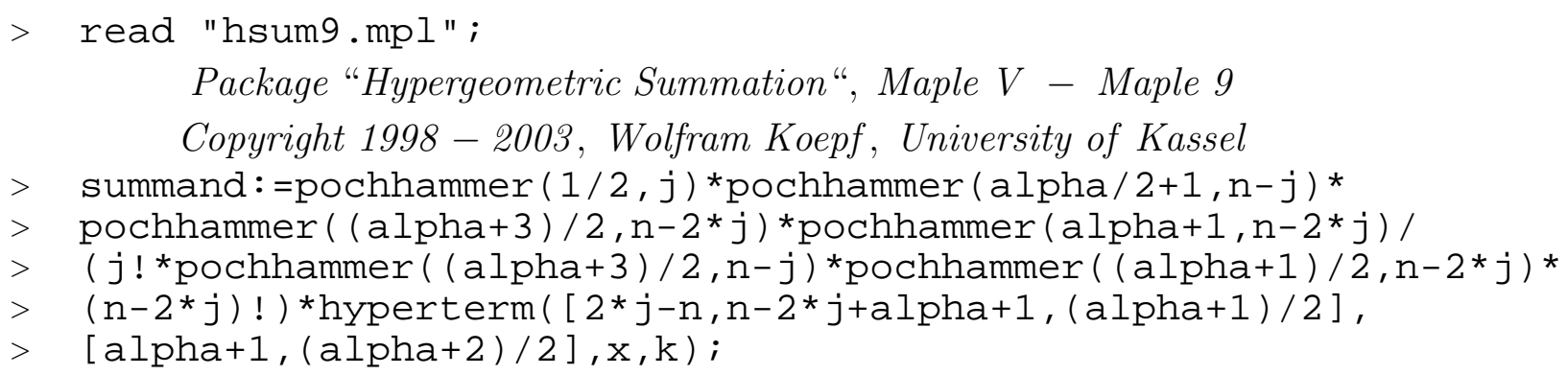


summand $:=\operatorname{pochhammer}\left(\frac{1}{2}, j\right)$ pochhammer $\left(\frac{\alpha}{2}+1, n-j\right)$ pochhammer $\left(\frac{\alpha}{2}+\frac{3}{2}, n-2 j\right)$ $\operatorname{pochhammer}(\alpha+1, n-2 j) \operatorname{pochhammer}(2 j-n, k)$

$\operatorname{pochhammer}(n-2 j+\alpha+1, k) \operatorname{pochhammer}\left(\frac{\alpha}{2}+\frac{1}{2}, k\right) x^{k} /(j !$

pochhammer $\left(\frac{\alpha}{2}+\frac{3}{2}, n-j\right)$ pochhammer $\left(\frac{\alpha}{2}+\frac{1}{2}, n-2 j\right)(n-2 j)$ !

pochhammer $(\alpha+1, k)$ pochhammer $\left.\left(\frac{\alpha}{2}+1, k\right) k !\right)$

By changing the order of summation, Zeilberger's algorithms yields the recurrence equation for the summand of the outer sum:

$>\mathrm{RE}:=$ sumrecursion (summand, j, $\mathrm{S}(\mathrm{k})$ );

$$
\begin{aligned}
& R E:=(k+1)(\alpha+1+k)(\alpha+3+2 k) \mathrm{S}(k+1) \\
& +x(\alpha+1+2 k)(-k+n)(n+\alpha+2+k) \mathrm{S}(k)=0
\end{aligned}
$$

Hence the sum is a multiple of

$>$ hyp:=hypergeom $([-\mathrm{n}, \mathrm{n}+2+\mathrm{alpha},(\mathrm{alpha}+1) / 2]$,

$>\quad[a l p h a+1,(a l p h a+3) / 2], x)$;

$$
\text { hyp }:=\operatorname{hypergeom}\left(\left[\frac{\alpha}{2}+\frac{1}{2},-n, n+2+\alpha\right],\left[\frac{\alpha}{2}+\frac{3}{2}, \alpha+1\right], x\right)
$$

We compute the initial value $S(k=0)$ by the same algorithm:

$>$ sumrecursion ( $\operatorname{subs}(k=0$, summand), j, init $(n))$;

$$
-(1+n) \operatorname{init}(1+n)+(n+2+\alpha) \operatorname{init}(n)=0
$$

Hence $S(0)$ is given as

$>\quad \mathrm{S} 0:=$ pochhammer $(2+\mathrm{alpha}, \mathrm{n}) / \mathrm{n} !$;

$$
S 0:=\frac{\text { pochhammer }(\alpha+2, n)}{n !}
$$

This proof goes from right to left, but there is no way to detect the right hand side from the left hand side. This part still needs Askey's and Gasper's ingenuity.

The clue of the Askey-Gasper identity is the fact that the hypergeometric function occurring in its right hand summand is a complete square by Clausen's identity

$$
{ }_{3} F_{2}\left(\begin{array}{c}
2 a, 2 b, a+b \\
2 a+2 b, a+b+1 / 2
\end{array} \mid x\right)={ }_{2} F_{1}\left(\begin{array}{c}
a, b \\
a+b+1 / 2
\end{array} \mid x\right)^{2} .
$$

Clausen's identity can also be proved by Zeilberger's algorithm. For this purpose, we write the right hand side as a Cauchy product, and an application of Zeilberger's algorithm leads to a first order recurrence for the outer summand which can be brought in closed form by the command

$>\quad$ Closedform (hyperterm $(\{a, b\},\{a+b+1 / 2\}, x, j) *$
$>\quad$ hyperterm $(\{a, b\},\{a+b+1 / 2\}, x, k-j), j, k) ;$

Hyperterm $\left([2 b, 2 a, b+a],\left[2 a+2 b, a+b+\frac{1}{2}\right], x, k\right)$ 


\section{Weinstein's Proof}

In the year 1989 Weinstein [Weinstein1991] succeeded with a proof of the Milin conjecture without using the Askey-Gasper result.

1989 WeInstein $\quad \begin{aligned} & \text { alternate proof of the de Branges } \\ & \text { theorem }\end{aligned}$

I would like to present Weinstein's proof in little more detail (see also my review 0743.30021 in Zentralblatt Mathematik), first, since it is impressively simple, and second, since the article [Weinstein1991] is not easily accessible.

Weinstein uses the following representation of the $t$-derivatives of the logarithmic coefficients $d_{k}(t)$ of the Loewner chain $\left(\zeta:=r e^{i \theta}, r \in(0,1)\right)$

$$
\begin{array}{cl}
\dot{d}_{k}(t) \stackrel{\text { (Cauchy formula) }}{=} \frac{1}{2 \pi} \int_{0}^{2 \pi} \frac{\dot{f}(\zeta, t)}{f(\zeta, t)} \frac{d \theta}{\zeta^{k}} \\
\stackrel{\frac{(6)}{2}}{=} \int_{0}^{2 \pi} p(\zeta, t) \frac{\zeta f^{\prime}(\zeta, t)}{f(\zeta, t)} \frac{d \theta}{\zeta^{k}} \\
=\quad \frac{1}{2 \pi} \int_{0}^{2 \pi} p(\zeta, t)\left(1+\sum_{j=1}^{\infty} j d_{j}(t) \zeta^{j}\right) \frac{d \theta}{\zeta^{k}} \\
=\quad \lim _{r \rightarrow 1} \frac{1}{2 \pi} \int_{0}^{2 \pi} p(\zeta, t)\left(1+\sum_{j=1}^{\infty} j d_{j}(t) \zeta^{j}\right) \frac{d \theta}{\zeta^{k}} .
\end{array}
$$

Let now $z \in \mathbb{D}$ and set $\varphi(z, t):=K^{-1}\left(e^{-t} K(z)\right)$, hence

$$
\frac{z}{(1-z)^{2}}=e^{t} \frac{\varphi}{(1-\varphi)^{2}} \quad(t \geq 0) \text {. }
$$

The mapping $\varphi: \mathbb{D} \times \mathbb{R}_{\geq 0} \rightarrow \mathbb{D}$ is a special Loewner family of bounded univalent functions whose image domains are the unit disk with a radial slit on the negative real axis that increases with $t$. Taking derivative in the defining equation (22) leads to the relation

$$
\dot{\varphi}=-\varphi \frac{1-\varphi}{1+\varphi}
$$

By de Branges' success Weinstein knew that the Milin conjecture is valid, hence he could easily try to prove the conjecture for all $n \in \mathbb{N}$ at the same time. Therefore he considered a generating function of the Milin expression (13). Because of the compactness of $S$ this generating function converges absolutely and locally uniformly in $\mathbb{D}$, and changing the order of summation yields

$$
\omega(z):=\sum_{n=1}^{\infty}\left(\sum_{k=1}^{n}(n+1-k)\left(\frac{4}{k}-k\left|d_{k}(0)\right|^{2}\right)\right) z^{n+1}=\frac{z}{(1-z)^{2}} \sum_{k=1}^{\infty}\left(\frac{4}{k}-k\left|d_{k}(0)\right|^{2}\right) z^{k} .
$$


Note that this procedure automatically generates the Koebe function as a factor! Using (22) and the fundamental theorem of calculus implies with $\varphi(z, \infty)=0$ and $\varphi(z, 0)=z$

$$
\omega(z)=\int_{0}^{\infty}-\frac{e^{t} \varphi}{(1-\varphi)^{2}} \frac{d}{d t}\left(\sum_{k=1}^{\infty}\left(\frac{4}{k}-k\left|d_{k}(t)\right|^{2}\right) \varphi^{k}\right) d t .
$$

Using the relations (21) and (23) gives after some further computations $\left(\zeta:=r e^{i \theta}, r \in(0,1)\right)$

$$
\omega(z)=\int_{0}^{\infty} \frac{e^{t} \varphi}{1-\varphi^{2}} \sum_{k=1}^{\infty} A_{k}(t) \varphi^{k} d t
$$

where

$$
A_{k}(t):=\lim _{r \rightarrow 1} \frac{1}{2 \pi} \int_{0}^{2 \pi} \operatorname{Re} p(\zeta, t)\left|1+2 \sum_{j=1}^{k} j d_{j}(t) \zeta^{j}-k d_{k}(t) \zeta^{k}\right|^{2} d \theta .
$$

If we now write

$$
W_{k}(z, t)=\frac{e^{t} \varphi^{k+1}}{1-\varphi^{2}}=: \sum_{n=k}^{\infty} \Lambda_{k}^{n}(t) z^{n+1}
$$

then finally

$$
\omega(z)=\sum_{n=1}^{\infty}\left(\int_{0}^{2 \pi} \sum_{k=1}^{\infty} \Lambda_{k}^{n}(t) A_{k}(t) d t\right) z^{n+1} .
$$

Remember that the Milin conjecture is equivalent to the fact that $\omega$ has non-negative coefficients. On the other hand Loewner's equation $(6), \operatorname{Re} p(\zeta, t)>0$, yields

$$
A_{k}(t) \geq 0 \quad(t \geq 0),
$$

so that the Milin conjecture follows if

$$
\Lambda_{k}^{n}(t) \geq 0 \quad(t \geq 0, \quad k, n \in \mathbb{N}) .
$$

This, again, is a positivity statement of a system of special real functions. These functions, however, are intimately related with the Koebe function and therefore with geometric function theory.

The range of $\varphi=K^{-1}\left(e^{-t} K\right)$ is the unit disk with a slit on the real axis. Since the mapping $(\gamma \in \mathbb{R})$

$$
h_{\gamma}(z):=\frac{z}{1-2 \cos \gamma \cdot z+z^{2}}
$$

maps the unit disk for $\gamma \neq 0(\bmod \pi)$ onto a domain which is slit on the real axis twice, we can also interpret $\varphi$ as the composition $\varphi=h_{\theta}^{-1}\left(e^{-t} h_{\gamma}\right)$ for a suitable pair $(\theta, \gamma)$, and a computation shows the relation

$$
\cos \gamma=\left(1-e^{-t}\right)+e^{-t} \cos \theta
$$

We therefore get

$$
\begin{aligned}
h_{\gamma}(z) & =e^{t} \cdot h_{\theta}(\varphi)=\frac{e^{t} \varphi}{1-\varphi^{2}}\left(\frac{1-\varphi^{2}}{1-2 \cos \theta \cdot \varphi+\varphi^{2}}\right) \\
& =\frac{e^{t} \varphi}{1-\varphi^{2}} \operatorname{Re} \frac{1+e^{i \theta} \varphi}{1-e^{i \theta} \varphi}=\frac{e^{t} \varphi}{1-\varphi^{2}}\left(1+2 \sum_{k=1}^{\infty} \varphi^{k} \cos k \theta\right) \\
& =\frac{e^{t} \varphi}{1-\varphi^{2}}+2 \sum_{k=1}^{\infty}\left(\sum_{n=1}^{\infty} \Lambda_{k}^{n}(t) z^{n+1}\right) \cos k \theta .
\end{aligned}
$$


Since

$$
\eta(z):=\frac{\sqrt{h_{\gamma}(z)}}{z}=\frac{1}{\sqrt{1-2 \cos \gamma \cdot z+z^{2}}}
$$

is the generating function of the Legendre polynomials, it follows from (27) and the addition theorem of the Legendre polynomials ([Laplace1782], see also [Legendre1789], [Heine1878]), an explicit representation of the form

$$
\eta(z)=\sum_{n=0}^{\infty} \nu_{n}^{2} z^{n}+2 \sum_{k=1}^{\infty}\left(\sum_{n=k}^{\infty} \frac{(n-k) !}{(n+k) !} \mu_{k n}^{2} z^{n}\right) \cos k \theta \quad\left(\nu_{n}, \mu_{k n} \in \mathbb{R} \quad(k, n \in \mathbb{N})\right)
$$

with obviously non-negative coefficients. After squaring this result, it follows from (28) that (26) is fulfilled, and therefore the Milin conjecture is true. This finishes Weinstein's proof.

Weinstein's proof uses the dynamic information of the Loewner differential equation in the form (25), i.e., he proves the fact that the Milin expressions (13) can be represented as time integrals with throughout non-negative integrands.

Note that Weinstein's paper has only four pages, but is written very densely, containing no single unnecessary word. This should be compared with my Zentralblatt review 0743.30021 of this paper which has also four pages, but which contains some historical remarks as well.

Undoubtedly, Bieberbach would have understood the proof of the Milin conjecture in Weinstein's form. The history of the proof, however, shows that a single researcher was not able to prove the conjecture, but the ideas of many people were necessary to collect the puzzle.

Especially

- the introduction of the dynamics of univalent functions and their describing differential equation by Loewner,

- the discovery of the relevance of weighted quadratic means by Robertson,

- the exponentiation of the logarithmic coefficients by Lebedev and Milin,

- as well as the systematic study of a special dynamic variant of the Milin conjecture by de Branges

eventually led to Weinstein's direct proof of the Milin conjecture by an application of the special Loewner chain generated by the Koebe function.

The Bieberbach conjecture has vastly influenced the research in geometric function theory, and de Branges' seminal discovery leaves a hole which may be bitterly missed by some researchers. The history of this conjecture, however, is a typical example how mathematical knowledge is generated.

\section{A Computer Algebra Proof of the Weinstein Theorem}

In [EZ1994], Ekhad and Zeilberger showed how the final part of Weinstein's proof version of the Bieberbach conjecture can be principally computerized. The main argument is the following: The coefficients of the function $\eta(z)$ given by

$$
\frac{1}{\sqrt{1-z\left(2 x^{2}+\left(1-z^{2}\right)(w+1 / w)\right)+z^{2}}}=\sum_{n=0}^{\infty} \sum_{k=0}^{n} \frac{(n-k) !}{(n+k) !}\left(1-x^{2}\right)^{k} C_{k, n}(x)\left(w^{k}+w^{-k}\right) z^{n}
$$


are polynomials $C_{k, n}(x) \in \mathbb{Q}[x]$ with rational coefficients. To prove that these form the squares of another system of polynomials

$$
D_{k, n}(x)^{2}=C_{k, n}(x)
$$

-which indeed is one of the major steps in Weinstein's proof-one should calculate the first polynomials $D_{k, n}(x)$ for $0 \leq k \leq n \leq 20$, and then "guess" a holonomic recurrence equation w.r.t. $n$, i.e., a homogeneous linear differential equation with polynomial coefficients $\sigma_{j} \in$ $\mathbb{Q}[k, n]$,

$$
\sigma_{2} D_{k, n+2}(x)+\sigma_{1} D_{k, n+1}(x)+\sigma_{0} D_{k, n}(x)=0,
$$

and to use linear algebra to find $\sigma_{0}, \sigma_{1}, \sigma_{2}$. With an own Maple implementation I was able to carry out this step. It turns out that

$$
(n-k+2) D_{k, n+2}(x)-(2 n+3) x D_{k, n+1}(x)+(n+k+1) D_{k, n}(x)=0
$$

and only the initial values for $0 \leq k \leq n \leq 6$ were necessary to find this equation.

Assume, $E_{k, n}(x)$ is the solution of (30) with the appropriate initial values. Then by another application of linear algebra this recurrence equation can be "squared", i.e. it is possible to calculate the recurrence equation $\mathcal{R}$ of third order valid for $E_{k, n}(x)^{2}$. This step can be accomplished by Maple's gfun packacke [SZ1994]. Then, finally, by an implementation of Zeilberger, using the so-called WZ method [WZ1992] one can show that $\mathcal{R}$ is valid for $C_{k, n}(x)$. This gives an a posteriori proof of the guessed (29). ${ }^{2}$

\section{The de Branges and the Weinstein Functions}

The question was posed to identify the Weinstein functions $\Lambda_{k}^{n}(t)$. Todorov [Todorov1992] and Wilf [Wilf1994] independently proved the surprising identity

$$
\dot{\tau}_{k}^{n}(t)=-k \Lambda_{k}^{n}(t) .
$$

This shows that the $t$-derivatives of the de Branges functions and the Weinstein functions essentially agree. In particular, the essential inequalities are the same.

We give an elementary proof of (31), see [KS1996]. Since by (24)

$$
W_{k}(z, t)=\frac{e^{t} \varphi^{k+1}}{1-\varphi^{2}}=\frac{e^{t} \varphi}{(1-\varphi)^{2}} \frac{1-\varphi}{1+\varphi} \varphi^{k}=K(z) \frac{1-\varphi}{1+\varphi} \varphi^{k}
$$

and

$$
W_{k+1}(z, t)=\varphi W_{k}(z, t)
$$

we have

$$
W_{k}(z, t)+W_{k+1}(z, t)=(1+\varphi) W_{k}(z, t)=K(z)(1-\varphi) \varphi^{k}=K(z) \varphi^{k}-K(z) \varphi^{k+1} .
$$

Taking derivative w.r.t. $t$, we get

$$
\begin{aligned}
\dot{W}_{k}(z, t)+\dot{W}_{k+1}(z, t) & =K(z) k \varphi^{k-1} \dot{\varphi}-K(z)(k+1) \varphi^{k} \dot{\varphi} \\
& =-k K(z) \frac{1-\varphi}{1+\varphi} \varphi^{k}+(k+1) K(z) \frac{1-\varphi}{1+\varphi} \varphi^{k+1} \\
& =(k+1) W_{k+1}(z, t)-k W_{k}(z, t)
\end{aligned}
$$

\footnotetext{
${ }^{2}$ The given proof differs from the argument of Ekhad's and Zeilberger's paper slightly and was first presented in my Zentralblatt review 0894.30013 of this paper where I corrected some minor inaccuracies.
} 
where we have used (23). Hence $W_{k}(z, t)$ satisfies the differential equation system of de Branges. Comparing the coefficients of $z^{n+1}$ one realizes that the same differential equation system is valid for $\Lambda_{k}^{n}(t)$. Checking the initial values proves the assertion (31).

The strong relation between the de Branges functions and the Koebe function can be also seen by their generating function w.r.t. $n$ [KS1996]:

$$
\begin{aligned}
\sum_{n=k}^{\infty} \tau_{k}^{n}(t) z^{n+1} & =B_{k}(z, t):=K(z) \varphi(z, t)^{k} \\
& =K(z)^{k+1} e^{-k t} F_{2}\left(\begin{array}{c}
k, k+1 / 2 \\
2 k+1
\end{array} \mid-4 K(z) e^{-t}\right) \\
& \left.=\sum_{n=k}^{\infty} e^{-k t}\left(\begin{array}{c}
n+k+1 \\
2 k+1
\end{array}\right){ }_{4} F_{3}\left(\begin{array}{c}
k+\frac{1}{2}, n+k+2, k, k-n \\
k+1,2 k+1, k+\frac{3}{2}
\end{array}\right) e^{-t}\right) z^{n+1}
\end{aligned}
$$

To obtain these results algorithmically, we would like to start with the proposed generating function $B_{k}(z, t)=K(z) \varphi^{k}(z, t)$, and compute its Taylor coefficients w.r.t $y=e^{-t}$, see [Koepf2003]. Therefore we consider the following general situation.

Given an expression $f(x)$ in the variable $x$, one would like to find the Taylor series

$$
f(x)=\sum_{k=0}^{\infty} A_{k} x^{k}
$$

i.e., a formula for the coefficient $A_{k}$. For example, if $f(x)=e^{x}$, then

$$
f(x)=\sum_{k=0}^{\infty} \frac{1}{k !} x^{k},
$$

hence $A_{k}=\frac{1}{k !}$. The main idea behind the FPS (Formal Power Series) algorithm [Koepf1992] is

- to compute a holonomic differential equation for $f(x)$, i.e., a homogeneous linear differential equation with polynomial coefficients,

- to convert the differential equation to a holonomic recurrence equation for $A_{k}$,

- and to solve the recurrence equation for $A_{k}$.

The above procedure is successful at least if $f(x)$ is a hypergeometric power series.

As a first application of the FPS algorithm, we compute the Taylor series of $w(z, y)^{k}=$ $\varphi(z,-\ln y)^{k}$, considered as function of the variable $y=e^{-t}$,

$$
w(z, y)^{k}=\frac{(4 y z)^{k}}{\left(1-z+\sqrt{1-2(1-2 y) z+z^{2}}\right)^{2 k}} .
$$

which turns out to be a hypergeometric power series: We load the package

$>$ read "FPS.mpl";

Package Formal Power Series, Maple $V-8$

Copyright 1995, Dominik Gruntz, University of Basel

Copyright 2002, Detlef Müller \& Wolfram Koepf, University of Kassel 
and enter

$$
\begin{aligned}
& >\text { assume }(z>0, z<1) \text {; interface ( } \text { h howassumed=0); } \\
& >\mathrm{w}:=4{ }^{\star} \mathrm{y}^{\star} \mathrm{z} /\left(1-\mathrm{z}+\operatorname{sqrt}\left(1-2 \star(1-2 * \mathrm{y}) \star \mathrm{z}+\mathrm{z}^{\wedge} 2\right)\right)^{\wedge} 2 \text {; } \\
& w:=\frac{4 y z}{\left(1-z+\sqrt{1-2 z+4 z y+z^{2}}\right)^{2}} \\
& >\mathrm{s}:=\operatorname{standardsum}\left(\mathrm{y}^{\wedge} \mathrm{k} * \operatorname{FPS}\left((\mathrm{w} / \mathrm{y})^{\wedge} \mathrm{k}, \mathrm{y}, \mathrm{j}\right)\right) \text {; }
\end{aligned}
$$

FPS/hypergeomRE: provided that $-1<=\min \left(-1,-2{ }^{*} \mathrm{k}-1\right)$

$$
s:=\sum_{j=0}^{\infty} \frac{y^{k}(-1)^{j} z^{(j+k)}\left(\frac{1}{(-1+z)^{2}}\right)^{(j+k)} \operatorname{pochhammer}(2 k, 2 j) y^{j}}{\operatorname{pochhammer}(2 k+1, j) j !}
$$

The computation gives the power series which can be brought into hypergeometric form:

$>$ simplify (Sumtohyper $(o p(1, s), j))$;

$$
y^{k} z^{k}\left(\frac{1}{(-1+z)^{2}}\right)^{k} \text { Hypergeom }\left(\left[k, k+\frac{1}{2}\right],[2 k+1],-\frac{4 z y}{(-1+z)^{2}}\right)
$$

This is (33). To obtain (34), i.e. the hypergeometric representation (19) of $\tau_{k}^{n}$, we multiply by $K(z)$, and apply the FPS procedure a second time, this time w.r.t. the variable $z$ :

$>\mathrm{ss}:=\operatorname{standardsum}\left(\operatorname{FPS}\left(\mathrm{z} /(1-\mathrm{z})^{\wedge} 2 * \mathrm{~s}, \mathrm{z}, \mathrm{i}\right)\right)$;

$$
\begin{aligned}
& \text { ss }:=\sum_{j=0}^{\infty} \\
& \quad\left(\sum_{i=0}^{\infty}\left(\frac{2(-1)^{j} y^{(j+k)} k \Gamma(2 k+2 j) \text { pochhammer }(2 k+2 j+2, i) z^{(i+1+j+k)}}{\Gamma(j+2 k)(j+2 k) j ! i !}\right)\right) \\
& >\quad \text { summand }:=\text { op }([1,1], \mathrm{ss}) ; \\
& \text { summand }:=\frac{2(-1)^{j} y^{(j+k)} k \Gamma(2 k+2 j) \text { pochhammer }(2 k+2 j+2, i) z^{(i+1+j+k)}}{\Gamma(j+2 k)(j+2 k) j ! i !} \\
& >\quad \text { summand }:=\operatorname{subs}(\mathrm{i}=\mathrm{n}-j-\mathrm{k}, \text { summand }) ; \\
& \text { summand }:=\frac{2(-1)^{j} y^{(j+k)} k \Gamma(2 k+2 j) \text { pochhammer }(2 k+2 j+2, n-j-k) z^{(n+1)}}{\Gamma(j+2 k)(j+2 k) j !(n-j-k) !} \\
& >\quad \text { result }:= \\
& \quad \text { convert }(\text { Sumtohyper }(\text { summand, } j), \text { binomial }) ;
\end{aligned}
$$

result $:=y^{k} z^{(n+1)} \operatorname{Hypergeom}\left(\left[k+2+n, k, k+\frac{1}{2},-n+k\right],\left[k+1,2 k+1, \frac{3}{2}+k\right], y\right)$ $\operatorname{binomial}(k+1+n, 2 k+1)$

The last computation gives (34), hence the hypergeometric representation (19) of $\tau_{k}^{n}(t)$.

An application of the same method automatically generates the hypergeometric representation for the Weinstein functions $\Lambda_{k}^{n}(t)$ directly from their defining generating function:

$$
\left.\Lambda_{k}^{n}(z, t)=e^{-k t}\left(\begin{array}{c}
k+n+1 \\
1+2 k
\end{array}\right){ }_{3} F_{2}\left(\begin{array}{c}
\frac{1}{2}+k, k-n, 2+k+n \\
1+2 k, \frac{3}{2}+k
\end{array}\right) e^{-t}\right) .
$$

Using (31) and (20) this finally yields the generating function (32). 


\section{Final Remarks}

The Maple packages used in this article can be downloaded from my web site http://www . mathematik.uni-kassel.de/ koepf/Publikationen.

As general references concerning the history of the Bieberbach conjecture I recommend the books of [Pommerenke1975], [Duren1977], [Duren1983], [Pommerenke1985], [BDDM1986], [Henrici1986] (pp. 605ff.) and [Gong1999].

\section{References}

[Aharonov1970] Aharonov, D.: On Bieberbach Eilenberg functions. Bull. Amer. Math. Soc. 76 (1970), 101-104.

[AskeyGasper1976] Askey, R. and Gasper, G.: Positive Jacobi polynomial sums II. Amer. J. Math. 98 (1976), 709-737.

[BDDM1986] Baernstein, A., Drasin, D., Duren, P. and Marden, A.: The Bieberbach conjecture. Proceedings of the Symposium on the Occasion of the Proof. Mathematical surveys and monographs 21, American Mathematical Society, Providence, R. I., 1986.

[Bieberbach1916] Bieberbach, L.: Über die Koeffizienten derjenigen Potenzreihen, welche eine schlichte Abbildung des Einheitskreises vermitteln. S.-B. Preuss. Akad. Wiss. 38 (1916), 940-955.

[deBranges1985] de Branges, L.: A proof of the Bieberbach conjecture. Acta Math. 154 (1985), $137-152$.

[Brickman1970] Brickman, L.: Extreme points of the set of univalent functions. Bull. Amer. Math. Soc. 76 (1970), 372-374.

[BMW1971] Brickman, L., MacGregor, T. H. and Wilken, D. R.: Convex hulls of some classical families of univalent functions, Trans. Amer. Math. Soc. 156 (1971), 91-107.

[Carathéodory1907] Carathéodory, C.: Über den Variabilitätsbereich der Koeffizienten von Potenzreihen, die gegebene Werte nicht annehmen. Math. Ann. 64 (1907), 95-115.

[Carathéodory 1911] Carathéodory, C.: Über den Variabilitätsbereich der Fourier'schen Konstanten von positiven harmonischen Funktionen. Rend. Circ. Mat. Palermo 32 (1911), 193217.

[Carathéodory 1912] Carathéodory, C.: Untersuchungen über die konformen Abbildungen von festen und veränderlichen Gebieten. Math. Ann. 72 (1912), 107-144.

[CS1960] Charzyński, Z. and Schiffer, M.: A new proof of the Bieberbach conjecture for the fourth coefficient. Arch. Rational Mech. Anal. 5 (1960), 187-193.

[CK1992] Collins, G. E. and Krandick, W.: An efficient algorithm for infallible polynomial complex roots isolation. In: Wang, Paul S. (Ed.): Proceedings of ISSAC'92 (1992), 189-194. 
[Dieudonné1931] Dieudonné, J.: Sur les fonctions univalentes. C. R. Acad. Sci. Paris 192 (1931), 1148-1150.

[Duren1977] Duren, P. L.: Coefficients of univalent functions. Bull. Amer. Math. Soc. 83 (1977), 891-911.

[Duren1983] Duren, P. L.: Univalent functions. Grundlehren der mathematischen Wissenschaften 259, Springer-Verlag, New York-Berlin-Heidelberg-Tokyo, 1983.

[EZ1994] Ekhad, S. B. and Zeilberger, D.: A high-school algebra, "Formal calculus", proof of the Bieberbach conjecture [after L. Weinstein]. Barcelo et al. (eds.): Jerusalem combinatorics '93: an international conference in combinatorics, May 9-17, 1993, Jerusalem, Israel. Providence, RI: American Mathematical Society. Contemp. Math. 178 (1994), $113-115$.

[FS1933] Fekete, M. and Szegö, G.: Eine Bemerkung über ungerade schlichte Funktionen. J. London Math. Soc. 8 (1933), 85-89.

[FitzGerald1972] FitzGerald, C. H.: Quadratic inequalities and coefficient estimates for schlicht functions. Arch. Rational Mech. Anal. 46 (1972), 356-368.

[FP1985] FitzGerald, C. H. and Pommerenke, Ch.: The de Branges Theorem on univalent functions. Trans. Amer. Math. Soc. 290 (1985), 683-690.

[Friedland1970] Friedland, S.: On a conjecture of Robertson. Arch. Rational Mech. Anal. 37 (1970), 255-261.

[GS1955] Garabedian, P. R. and Schiffer, M.: A proof of the Bieberbach conjecture for the fourth coefficient. J. Rational Mech. Anal. 4 (1955), 427-465.

[Gautschi1986] Gautschi, W.: Reminiscences of my involvement in de Branges's proof of the Bieberbach conjecture. In: Baernstein, Drasin, Duren and Marden (Eds): The Bieberbach conjecture (West Lafayette, Ind., 1985), Math. Surveys Monogr. 21, Amer. Math. Soc., Providence, RI (1986), 205-211.

[Gong1999] Gong, Sheng: The Bieberbach conjecture, Studies in Advanced Mathematics Vol. 12, American Mathematical Society, Providence, R.I., 1999.

[Gronwall1914] Gronwall, T. H.: Some remarks on conformal representation. Ann. of Math. 16 (1914-1915), 72-76.

[Grunsky1939] Grunsky, H.: Koeffizientenbedingungen für schlicht abbildende meromorphe Funktionen. Math. Z. 45 (1939), 29-61.

[Hamilton1988] Hamilton, D. H.: Extremal boundary problems. Proc. London Math. Soc. (3) 56 (1988), 101-113.

[Hayman1955] Hayman, W. K.: The asymptotic behaviour of $p$-valent functions. Proc. London Math. Soc. (3) 5 (1955), 257-284.

[HH1986] Hayman, W. K. and Hummel, J. A.: Coefficients of powers of univalent functions. Complex Variables 7 (1986), 51-70. 
[Heine1878] Heine, E.: Handbuch der Kugelfunctionen, Theorie und Anwendungen. Reimer, Berlin, 1878.

[Henrici1986] Henrici, P.: Applied and Computational Complex Analysis, Vol. 3: Discrete Fourier Analysis - Cauchy Integrals - Construction of Conformal maps - Univalent Functions, John Wiley \& Sons, New York, 1986.

[Horowitz1978] Horowitz, D.: A further refinement for coefficient estimates of univalent functions. Proc. Amer. Math. Soc. 71 (1978), 217-221.

[Hummel1976] Hummel, J. A.: A variational method for Gelfer functions. J. Analyse Math. 30 (1976), 271-280.

[Koebe1907] Koebe, P.: Über die Uniformisierung beliebiger analytischer Kurven. Nachr. Kgl. Ges. Wiss. Göttingen, Math-Phys. Kl. (1907), 191-210.

[Koebe1909] Koebe, P.: Über die Uniformisierung der algebraischen Kurven durch automorphe Funktionen mit imaginärer Substitutionsgruppe. Nachr. Kgl. Ges. Wiss. Göttingen, Math-Phys. Kl. (1909), 68-76.

[Koebe1912] Koebe, P.: Über eine neue Methode der konformen Abbildung und Uniformisierung. Nachr. Kgl. Ges. Wiss. Göttingen, Math-Phys. Kl. (1912), 844-848.

[Koepf1986] Koepf, W.: On nonvanishing univalent functions with real coefficients, Math. Z. 192 (1986), 575-579.

[Koepf1987] Koepf, W.: Extrempunkte und Stützpunkte in Familien nichtverschwindender schlichter Funktionen, Complex Variables 8 (1987), 153-171.

[Koepf1992] Koepf, W.: Power series in Computer Algebra. J. Symb. Comp. 13 (1992), 581603.

[Koepf1998] Koepf, W.: Hypergeometric Summation. Vieweg, Braunschweig/Wiesbaden, 1998.

[Koepf2003] Koepf, W.: Power series, Bieberbach conjecture and the de Branges and Weinstein functions. Proceedings of ISSAC 2003, Philadelphia. J. R. Sendra (Ed.), ACM, New York, 2003, 169-175.

[KS1996] Koepf, W. and Schmersau, D.: On the de Branges theorem. Complex Variables 31 (1996), 213-230.

[Laplace1782] Laplace, P.-S.: Théorie des attractions des sphéroïdes et de la figure des planètes. Mémoires de l'Academie Royale des Sciences de Paris (1782), 113-196.

[LM1965] Lebedev, N. A. and Milin, I. M.: An inequality. Vestnik Leningrad Univ. 20 (1965), 157-158 (Russian).

[Leeman1976] Leeman, G. B.: The seventh coefficient of odd symmetric univalent functions. Duke Math. J. 43 (1976), 301-307.

[Legendre1789] Legendre, A.-M.: Suite des recherches sur la figure des planètes, Mémoires de l'Academie Royale des Sciences de Paris (1789), 372-454. 
[Leung1978] Leung, Y.: Successive coefficients of starlike functions. Bull. London Math. Soc. 10 (1978), 193-196.

[Leung1979] Leung, Y.: Robertson's conjecture on the coefficients of close-to-convex functions. Proc. Amer. Math. Soc. 76 (1979), 89-94.

[Littlewood1925] Littlewood, J. E.: On inequalities in the theory of functions. Proc. London Math. Soc. (2) 23 (1925), 481-519.

[LP1932] Littlewood, J. E. and Paley, R. E. A. C.: A proof that an odd schlicht function has bounded coefficients. J. London Math. Soc. 7 (1932), 167-169.

[Loewner1917] Löwner, K.: Untersuchungen über die Verzerrung bei konformen Abbildungen des Einheitskreises $|z|<1$, die durch Funktionen mit nichtverschwindender Ableitung geliefert werden. S.-B. Verh. Sächs. Ges. Wiss. Leipzig 69 (1917), 89-106.

[Loewner1923] Löwner, K.: Untersuchungen über schlichte konforme Abbildungen des Einheitskreises I. Math. Ann. 89 (1923), 103-121.

[Milin1965] Milin, I. M.: Estimation of coefficients of univalent functions. Dokl. Akad. Nauk SSSR 160 (1965), 769-771 (Russian) = Soviet Math. Dokl. 6 (1965), 196-198.

[Milin1967] Milin, I. M.: On the coefficients of univalent functions. Dokl. Akad. Nauk SSSR 176 (1967), 1015-1018 (Russian) = Soviet Math. Dokl. 8 (1967), 1255-1258.

[Milin1971] Milin, I. M.: Univalent functions and orthonormal systems. Izdat. "Nauka", Moskau, 1971 (Russian). English Translation: Amer. Math. Soc., Providence, R. I., 1977.

[Milin1984] Milin, I. M.: De Branges' proof of the Bieberbach conjecture (Russian), Preprint (1984).

[Nehari1970] Nehari, Z.: On the coefficients of Bieberbach-Eilenberg functions. J. Analyse Math. 23 (1970), 297-303.

[Nehari1974] Nehari, Z.: A proof of $\left|a_{4}\right| \leq 4$ by Loewner's method. In: Proceedings of the Symposium on Complex Analysis, Canterbury, 1973 (edited by J. Clunie and W. K. Hayman), London Math. Soc. Lecture Note Series 12, Cambridge University Press, 1974, 107-110.

[Nevanlinna1920] Nevanlinna, R.: Über die konforme Abbildung von Sterngebieten. Översikt av Finska Vetenskaps-Soc. Förh. 63(A), Nr. 6 (1920-1921), 1-21.

[Ozawa1969a] Ozawa, M.: On the Bieberbach conjecture for the sixth coefficient. Kōdai Math. Sem. Rep. 21 (1969), 97-128.

[Ozawa1969b] Ozawa, M.: An elementary proof of the Bieberbach conjecture for the sixth coefficient. Kōdai Math. Sem. Rep. 21 (1969), 129-132.

[Pederson1968] Pederson, R. N.: A proof of the Bieberbach conjecture for the sixth coefficient. Arch. Rational. Mech. Anal. 31 (1968), 331-351. 
[PS1972] Pederson, R. N. and Schiffer, M.: A proof of the Bieberbach conjecture for the fifth coefficient. Arch. Rational. Mech. Anal. 45 (1972), 161-193.

[Pommerenke1975] Pommerenke, Ch.: Univalent functions. Vandenhoeck und Ruprecht, Göttingen-Zürich, 1975.

[Pommerenke1985] Pommerenke, Ch.: The Bieberbach Conjecture. Mathematical Intelligencer 7 (2) (1985), 23-25.

[Reade1955] Reade, M. O.: On close-to-convex univalent functions. Mich. Math. J. 3 (1955), $59-62$.

[Robertson1936] Robertson, M. S.: On the theory of univalent functions. Ann. of Math. 37 (1936), 374-408.

[Rogosinski1932] Rogosinski, W.: Über positive harmonische Entwicklungen und typisch-reelle Potenzreihen. Math. Z. 35 (1932), 93-121.

[SZ1994] Salvy, B. and Zimmermann, P.: GFUN: A Maple package for the manipulation of generating and holonomic functions in one variable. ACM Transactions on Mathematical Software 20 (1994), 163-177.

[Schiffer1938] Schiffer, M.: A method of variation within the family of simple functions. Proc. London Math. Soc. 44 (1938), 432-449.

[Study1913] Study, E.: Vorlesungen über ausgewählte Gegenstände der Geometrie, 2. Heft: Konforme Abbildung einfach-zusammenhängender Bereiche. Teubner-Verlag, LeipzigBerlin, 1913.

[Todorov1992] Todorov, P. G.: A simple proof of the Bieberbach conjecture. Bull. Cl. Sci., VI. Sér., Acad. R. Belg. 312 (1992), 335-356.

[Weinstein1991] Weinstein, L.: The Bieberbach conjecture. Internat. Math. Res. Notices 5 (1991), 61-64.

[Wilf1994] Wilf, H.: A footnote on two proofs of the Bieberbach-de Branges Theorem. Bull. London Math. Soc. 26 (1994), 61-63.

[WZ1992] Wilf, H. and Zeilberger, D.: An algorithmic proof theory for hypergeometric (ordinary and “ $q$ ") multisum/integral identities. Invent. Math. 103 (1992), 575-634.

[Zeilberger1990] Zeilberger, D.: A fast algorithm for proving terminating hypergeometric identities. Discrete Math. 80 (1990), 207-211. 
Wolfram Koepf: Bieberbach's Conjecture, the de Branges and Weinstein Functions and the Askey-Gasper Inequality

Contact address: Wolfram Koepf, University of Kassel, Department of Mathematics, HeinrichPlett-Str. 40, 34132 Kassel, Germany

Tel: +495618044207

Fax: +495618044646

E-mail: koepf@mathematik.uni-kassel.de

Key Words: Bieberbach conjecture, Robertson conjecture, Milin conjecture, convex functions, starlike functions, close-to-convex functions, Grunsky inequalities, Schiffer variation, support points, extreme points, Loewner differential equation, Loewner theory, Lebedev-Milin inequalities, de Branges theorem, de Branges functions, Weinstein functions, hypergeometric functions, generalized hypergeometric series, Askey-Gasper inequality, Askey-Gasper identity, Legendre addition theorem, FPS algorithm, Zeilberger algorithm, Maple, symbolic computation, computer algebra

AMS 2000 Classification: 30C50, 30C35, 30C45, 30C80, 33C20, 33C45, 33F10, 68W30 